\title{
gn
}

\section{Inhomogeneous Phase Formation on the Border of Itinerant Ferromagnetism}

\author{
G. J. Conduit, ${ }^{1, *}$ A. G. Green, ${ }^{2}$ and B. D. Simons ${ }^{1}$ \\ ${ }^{1}$ Cavendish Laboratory, 19, J.J. Thomson Avenue, Cambridge, CB3 OHE. United Kingdom \\ ${ }^{2}$ School of Physics and Astronomy, North Haugh, St Andrews, Fife, KY16 9SS. United Kingdom
}

(Received 7 June 2009; published 9 November 2009)

\begin{abstract}
A variety of analytical techniques suggest that quantum fluctuations lead to a fundamental instability of the Fermi liquid that drives ferromagnetic transitions first order at low temperatures. We present both analytical and numerical evidence that, driven by the same quantum fluctuations, this first order transition is preempted by the formation of an inhomogeneous magnetic phase. This occurs in a manner that is closely analogous to the formation of the inhomogeneous superconducting Fulde-Ferrel-LarkinOvchinnikov state. We derive these results from a field-theoretical approach supplemented with numerical quantum Monte Carlo simulations.
\end{abstract}

Many metallic magnetic materials display second order ferromagnetic phase transitions. In these itinerant ferromagnetic systems the transition temperature can be tuned using external parameters such as doping and pressure. Hertz realized that tuning such a transition to zero temperature could give rise to a new type of critical phenomenon, for which he coined the term quantum criticality [1]. This led to a tremendous experimental and theoretical effort that has had some notable successes with the universal scaling predicted for the quantum critical regime being seen in a handful of materials [2]. However, in the majority of itinerant ferromagnetic systems, new behavior intervenes before the transition temperature can be tuned to zero. In many cases, such as $\mathrm{ZrZn}_{2}$ [3], $\mathrm{UGe}_{2}$ [4], $\mathrm{MnSi}$ [5], and $\mathrm{CoS}_{2}$ [6], the second order transition becomes first order before the quantum critical point is reached. Moreover, materials such as $\mathrm{ZrZn}_{2}$ [3], $\mathrm{UGe}_{2}$ [7], $\mathrm{Ca}_{3} \mathrm{Ru}_{2} \mathrm{O}_{7}$ [8], $\mathrm{NbFe}_{2}$ [9], and $\mathrm{Sr}_{3} \mathrm{Ru}_{2} \mathrm{O}_{7}$ [10] all display unusual behavior in the vicinity of the putative quantum critical point that go beyond a first order transition.

This failure to find a naked quantum critical point has lead to speculation that it represents a fundamental principle [11]. Diagrammatic calculations that extend beyond the standard Moriya-Hertz-Millis theory of itinerant quantum criticality [1] suggest a breakdown of the Landau expansion around the quantum critical point $[12,13]$. This raises the question of how to connect these calculations to the second order perturbation approach [14] that accounts for all orders of vacuum scattering amplitude, and predicts a first order ferromagnetic transition at low temperature.

This Letter is divided into two parts: In the first we show that, when a linearization of the electron dispersion about the Fermi surface is permissible, with increasing repulsive interaction strength the system first forms an inhomogeneous magnetic phase prior to the transition into a uniform ferromagnetic phase. These results, which mirror the theory of the inhomogeneous superconducting Fulde-FerrelLarkin-Ovchinnikov (FFLO) state [15], are derived using a field-theoretical approach [16] that reveals how quantum fluctuations self-consistently stabilize a textured state that is not favored at the mean-field level, and also resolves the connection between the second order perturbation theory approach and the nonanalyticities that appear in extensions to the Moriya-Hertz-Millis theory [12]. Secondly, we describe quantum Monte Carlo (QMC) simulations that support our analytics.

In metals, the long-range component of the Coulomb interaction is screened. We therefore take as our starting point a free electron system interacting through a contact (Hubbard-like) repulsive interaction, $g \delta^{3}(\mathbf{r})$ [1]. The corresponding partition function may be expressed as a fermionic coherent state path integral, $Z=\operatorname{Tr} e^{-\beta(\hat{H}-\mu \hat{N})}=$ $\int \mathcal{D} \psi e^{-S}$, with the action

$$
S=\int \sum_{\sigma= \pm} \bar{\psi}_{\sigma}\left(\partial_{\tau}+\hat{\zeta}\right) \psi_{\sigma}+\int g \bar{\psi}_{+} \bar{\psi}_{-} \psi_{-} \psi_{+} .
$$

Here $\int \equiv \int_{0}^{\beta} d \tau \int d^{3} x, \hat{\zeta}_{\hat{\mathbf{k}}}=\epsilon_{\hat{\mathbf{k}}}-\mu$, with a general dispersion $\epsilon_{\hat{\mathbf{k}}}$, and $\mu$ represents the chemical potential. To develop an effective Landau theory of the magnetic transition, Hertz introduced a scalar Hubbard-Stratonovich decoupling of the interaction in the spin channel [1]. However, this form of decoupling neglects the potential impact of soft transverse field fluctuations that are responsible for driving the second order transition first order and in turn promote the instability towards inhomogeneous phase formation. Therefore, we will introduce a general Hubbard-Stratonovich decoupling which incorporates fluctuations in all of the spin $\boldsymbol{\phi}$ and charge $\rho$ sectors [17]. To simplify the analysis it is useful to transform to a rotating basis with pitch vector $\mathbf{q}$ in which the spin spiral would adopt a uniform magnetization and to gauge transform the fermions according to $\psi \mapsto e^{i \mathbf{q} \cdot \mathbf{r} \sigma_{z} / 2} \psi$. Finally, after integrating over the fermion degrees of freedom one obtains $\mathcal{Z}=\int \mathcal{D} \rho \mathcal{D} \phi e^{-S}$ where,

$$
S=\int g\left(\boldsymbol{\phi}^{2}-\rho^{2}\right)-\operatorname{Tr} \ln \left[\partial_{\tau}+\hat{\zeta}_{\mathbf{k}+\sigma_{z} \mathbf{q} / 2}+g \rho-g \boldsymbol{\sigma} \cdot \boldsymbol{\phi}\right] .
$$


At this stage, we could subject the action to a saddle point analysis to determine the mean-field values of $\rho$ and $\boldsymbol{\phi}$. However, fluctuations in $\rho$ and $\boldsymbol{\phi}$ renormalize these equations, changing the order of the transition into the uniform phase [16]. We therefore expand the action to quadratic order in fluctuations of $\rho$ and $\phi$ around their putative spatially homogeneous mean-field values $\rho_{0}$ and $\mathbf{m}=\left(m_{\perp}, 0, m_{\|}\right)$, with $m_{\perp}$ representing the spiral vector and $m_{\|}$the constant component. Integrating over the fluctuating fields yields the free energy,

$$
\begin{aligned}
\beta F= & \beta V g\left(m^{2}-\rho_{0}^{2}\right) \\
& -\operatorname{Tr} \ln \left(\partial_{\tau}+\hat{\zeta}_{\mathbf{k}+\sigma_{z} \mathbf{q} / 2}+g \rho_{0}-g \boldsymbol{\sigma} \cdot \mathbf{m}\right) \\
& +\operatorname{Tr} g \hat{\Pi}^{+-}+\frac{1}{2} \operatorname{Tr} g^{2}\left(\hat{\Pi}^{+-} \hat{\Pi}^{-+}-\hat{\Pi}^{++} \hat{\Pi}^{--}\right),
\end{aligned}
$$

Here $\hat{\Pi}^{s s^{\prime}}=\hat{G}^{s} \hat{G}^{s^{\prime}}$ denotes the Lindhard function, $\hat{G}^{ \pm}=$ $\left(\partial_{\tau}+\epsilon_{\hat{\mathbf{k}}, \mathbf{q}}^{ \pm}+g \rho_{0}-\mu\right)^{-1}$, and $\epsilon_{\mathbf{k}, \mathbf{q}}^{ \pm}$represents the energy of the electrons in plane-wave states with momentum $\mathbf{k}$ and spin-up or down relative to the mean-field spiral, $2 \epsilon_{\mathbf{k}, \mathbf{q}}^{ \pm}=\epsilon_{\mathbf{k}+(\mathbf{q} / 2)}+\epsilon_{\mathbf{k}-(\mathbf{q} / 2)} \pm\left[\left(\epsilon_{\mathbf{k}+(\mathbf{q} / 2)}-\epsilon_{\mathbf{k}-(\mathbf{q} / 2)}+\right.\right.$ $\left.\left.2 g m_{\|}\right)^{2}+\left(2 g m_{\perp}\right)^{2}\right]^{1 / 2}$.

Finally, carrying out the remaining Matsubara frequency summations, one obtains the free energy

$$
\begin{aligned}
F= & \sum_{\mathbf{k}, s} \epsilon_{\mathbf{k}, \mathbf{q}}^{s} n\left(\epsilon_{\mathbf{k}, \mathbf{q}}^{s}\right)+\frac{2 k_{F} a}{\pi \nu V} N_{\mathbf{q}}^{+} N_{\mathbf{q}}^{-} \\
& -2\left(\frac{2 k_{F} a}{\pi \nu V}\right)^{2} \sum_{\mathbf{k}} \int d \epsilon^{+} d \epsilon^{-} \frac{\eta_{\mathbf{q}}^{+}\left(\mathbf{k}, \epsilon^{+}\right) \eta_{\mathbf{q}}^{-}\left(-\mathbf{k}, \epsilon^{-}\right)}{\epsilon^{+}+\epsilon^{-}} \\
& +2\left(\frac{2 k_{F} a}{\pi \nu V}\right)^{2} \sum_{\mathbf{k}_{1,2,3,4}}^{\prime} \frac{n\left(\epsilon_{\mathbf{k}_{1}, \mathbf{q}}^{+}\right) n\left(\epsilon_{\mathbf{k}_{2}, \mathbf{q}}^{-}\right)}{\epsilon_{\mathbf{k}_{1}, \mathbf{q}}^{+}+\epsilon_{\mathbf{k}_{2}, \mathbf{q}}^{-}-\epsilon_{\mathbf{k}_{3}, \mathbf{q}}^{+}-\epsilon_{\mathbf{k}_{4}, \mathbf{q}}^{-}},
\end{aligned}
$$

with $\quad N_{\mathbf{q}}^{s}=\sum_{\mathbf{k}} n\left(\epsilon_{\mathbf{k}, \mathbf{q}}^{s}\right), \quad \eta_{\mathbf{q}}^{s}(\mathbf{k}, \epsilon)=\sum_{\mathbf{k}^{\prime}} n\left(\epsilon_{\mathbf{k}^{\prime}+\mathbf{k} / 2, \mathbf{q}}^{s}\right) \times$ $\left[1-n\left(\epsilon_{\mathbf{k}^{\prime}-\mathbf{k} / 2, \mathbf{q}}^{s}\right)\right] \delta\left(\epsilon-\epsilon_{\mathbf{k}^{\prime}+\mathbf{k} / 2, \mathbf{q}}^{s}+\epsilon_{\mathbf{k}^{\prime}-\mathbf{k} / 2, \mathbf{q}}^{s}\right)$ is the density of particle-hole pairs with spin $s$ (and $n(\epsilon)=[1+$ $\left.e^{\beta(\epsilon-\mu)}\right]^{-1}$ is the Fermi distribution). We have used the standard regularization setting $g \mapsto \frac{2 k_{F} a}{\pi \nu}-\frac{2}{V}\left(\frac{2 k_{F} a}{\pi \nu}\right)^{2} \times$ $\sum_{\mathbf{k}_{3,4}}^{\prime}\left(\epsilon_{\mathbf{k}_{1}, \mathbf{q}}^{+}+\epsilon_{\mathbf{k}_{2}, \mathbf{q}}^{-}-\epsilon_{\mathbf{k}_{3}, \mathbf{q}}^{+}-\epsilon_{\mathbf{k}_{4}, \mathbf{q}}^{-}\right)^{-1}[18]$, in order to remove the unphysical ultraviolet divergence of the contact interaction. The prime indicates that the summation is subject to the momentum conservation $\mathbf{k}_{1}+\mathbf{k}_{2}=\mathbf{k}_{3}+$ $\mathbf{k}_{4}$. This regularization allows us to characterize the strength of the interaction through the dimensionless parameter $k_{F} a$, where $k_{F}$ denotes the Fermi wave vector, $a$ is the $s$-wave scattering length, and $\nu$ is the density of states at the Fermi surface. A similar expression was derived in the homogeneous case in Ref. [14] using second order perturbation theory. Equation (2) - justified on the basis of our field-theoretical analysis - can be thought of as a generalization to a spiral background.

Uniform magnetization.-We first consider the implications of the fluctuation corrections on the magnetic phase diagram for a free particle dispersion $\epsilon_{\mathbf{k}}=k^{2} / 2 m^{*}$ without accounting for spatial modulation. By minimiz- ing the free energy Eq. (2) with respect to the magnetization $\mathbf{m}$ we recover the phase behavior. In this case one finds that fluctuations drive the second order ferromagnetic transition first order below the tricritical point temperature $T_{T} \approx 0.2 T_{F}$, where $T_{F}$ is the Fermi temperature (see Fig. 1) [14]. Focusing on $T=0$, substituting the low energy form of the particle-hole density of states, $\eta_{\mathbf{q}=\mathbf{0}}^{ \pm}(\mathbf{k}, \boldsymbol{\epsilon})=\epsilon \theta\left(k k_{F}^{ \pm}-k^{2} / 2-\epsilon\right) / 2 \pi k, \quad$ where $\quad k_{F}^{ \pm}=$ $\sqrt{2 m^{*}\left(\mu \pm 2 k_{F} a m / \pi \nu\right)}$, into the fluctuation correction to the free energy, and expanding in powers of magnetization, one recovers a singular term of order $m^{4} \ln m^{2}$ arising from particle-hole excitations with momentum near to $2 k_{F}$, the same nonanalyticity as that found diagrammatically in Refs. [12]. A finite magnetization increases the phase space available for the formation of virtual intermediate particle-hole excitations, and this enhancement ultimately drives the ferromagnetic transition first order.

Textured magnetization.-Quantum fluctuations also lead to spatial modulation of the magnetic order and further reconstruction of the magnetic phase diagram. If we anticipate that the transition into the spatially modulated phase is continuous and leads to a planar spiral (i.e. $m_{\|}=0$ ), we may determine the phase boundary by expanding Eq. (2) to quadratic order in $m_{\perp}$, and then search for the $\mathbf{q}$ and $k_{F} a$ where the coefficient of $m_{\perp}^{2}$ first turns negative. The resulting phase diagram in Fig. 1 reveals that a textured phase preempts the transition into the uniform phase, and, in particular, the phase terminates at the tricritical point.

To understand this phase behavior, it is instructive to look at the Ginzburg-Landau expansion in the vicinity of the tricritical point [19]. For a quadratic dispersion, the magnetization and spiral wave vector enter the single elec-

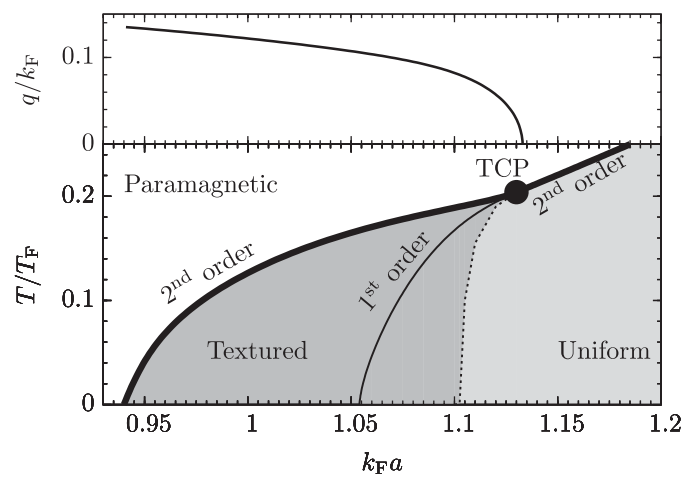

FIG. 1. The lower graph shows the phase diagram consisting of paramagnetic, uniform and textured ferromagnetic phases. The thick solid locus of continuous transitions from the paramagnet into the textured phase and uniform ferromagnetic phase are determined from analysis of Eq. (2). The anticipated transition or crossover between the textured and uniform phase (shown dashed) requires expansion of Eq. (2) to higher order than that carried out here. The thin line shows where the first order transition from paramagnet to ferromagnet takes place if the magnetization were restricted to be uniform. The upper graph shows the magnitude of the wave vector $\mathbf{q}$ along the upper modulated phase boundary. 
tron energy $\epsilon_{\mathbf{k}, \mathbf{q}}^{ \pm}$in the combination $(\mathbf{k} \cdot \mathbf{q})^{2}+\left(4 \pi a m_{\perp}\right)^{2}$, where we have restricted attention to a planar spiral since (as we will see below) it always has lower energy than a conical spiral at zero magnetic field. Upon linearizing the electron energy at the Fermi surface, $q^{2}$ and $m_{\perp}^{2}$ enter the free energy in the same way (up to the angular factors that accompany $\mathbf{q}$ and which are integrated over). Therefore, the spatial modulation enters as if it where a direction dependent magnetization. As a consequence, the coefficient of $m_{\perp}^{4}$ in a Ginzburg-Landau expansion is proportional to that of $q^{2} m_{\perp}^{2}$ : i.e., $\quad F=\left(\alpha+\beta k_{F}^{2} q^{2} / 24 \pi^{2} a^{2}\right) m_{\perp}^{2}+$ $\beta m_{\perp}^{4}+\gamma m_{\perp}^{6}+\cdots$, where $\alpha, \beta$, and $\gamma$ are the same functions of $T$ and $k_{F} a$ as in the homogeneous case. When $\beta$ becomes negative and a first order transition becomes favorable, the $q^{2} m_{\perp}^{2}$-term also becomes negative favoring a spatial modulation that emerges from the tricritical point. This is exactly the same situation as in the FFLO state [15]. Whether or not this transition is continuous is, in general, controlled by nonuniversal parameters that depend on details of the dispersion. Finally, we justify the assumption that the spin spiral is planar by noticing that, for a conical spiral with $m_{\|}$nonzero and uniform, there would be an additional contribution to the Landau free energy of $\alpha m_{\|}^{2}$. However, the instability to a textured phase develops at an optimal $\mathbf{q}$ where $\alpha$ remains positive so the planar spiral is favored over a conical spiral.

To assess the validity of the perturbative scheme, we now turn now to the numerical quantum Monte Carlo analysis of the Stoner Hamiltonian (1) making use of the CASINO program [20]. These methods are based upon optimizing a trial wave function and are restricted to zero temperature. Our approach is a refinement of that used in previous studies of itinerant ferromagnetism [21]. The variational wave function used in our simulation, $\psi=$ $D e^{-J}$, is a product of a Slater determinant, $D$, that takes account of the Fermion statistics and occupation of single particle orbitals, and a Jastrow factor, $J$, that accounts for electron correlations.

The Slater determinant consists of plane-wave spinor orbitals containing both spin-up and spin-down electrons, $D=\operatorname{det}\left(\left\{\psi_{\mathbf{k} \in k_{\dagger}}, \bar{\psi}_{\mathbf{k} \in k_{\downarrow}}\right\}\right)$. Although the Slater determinant is not an eigenstate of total spin, when separated into its constituent spin basis states, those with low spin have a greater multiplicity than those with high spin. Therefore, to a good approximation, the spin is set by $S_{\text {tot }}^{z}$. In the case of uniform magnetization, for computational efficiency, we factorize the Slater determinant into an up and a down-spin determinant [20]. The spin textured phase is described by noncollinear spins, which have only recently been studied within the variational Monte Carlo (VMC) method [22]. These studies lead us to describe a planar spin spiral with a trial wave function that contains the spinors $\psi_{\mathbf{k}}=$ $e^{i \mathbf{q} \cdot \mathbf{r} / 2}\left(e^{i \mathbf{k} \cdot \mathbf{r}}, e^{-i \mathbf{k} \cdot \mathbf{r}}\right) \quad$ and $\quad \bar{\psi}_{\mathbf{k}}=e^{-i \mathbf{q} \cdot \mathbf{r} / 2}\left(-e^{i \mathbf{k} \cdot \mathbf{r}}, e^{-i \mathbf{k} \cdot \mathbf{r}}\right)$ which explicitly fix the spin spiral orientation. The ground state energy was estimated from QMC runs at $q / k_{F}=0$, 0.2 , and 0.4 ; at $q=0$ this would recover the factorized form for the Slater determinant employed in the uniform case. Our simulations are carried out in a unit cell with periodic boundary conditions commensurate with the pitch of the spiral.

The Jastrow factor, J, accounts for electron-electron correlations. It consists of polynomial and plane-wave expansions in the electron-electron separation [23]. To further optimize the wave function, the Slater determinant orbitals were evaluated at quasiparticle positions related to the electrons through a polynomial backflow function [24]. In the spiral case, the Jastrow factor is restricted to be spin independent to maintain the spin spiral orientation and the wave function antisymmetry. In the uniform case, the wave function optimization was performed in two steps using VMC and diffusion Monte Carlo, whereas only VMC calculations were performed for the textured state. To model the repulsive contact interaction between the electrons we employ the modified Pöschl-Teller potential [25] which has smooth edges so that the QMC configurations can sample it faithfully [26].

First, constraining the magnetization to be spatially uniform, an estimate of the ground state magnetization for different interaction strengths recovers a first order phase transition into the itinerant magnetic phase (Fig. 2). Although the QMC calculation reproduces the predicted phase behavior of the theory, the small but significant discrepancy between the critical values of $k_{F} a$ reflects the limitations of perturbation theory in the regime of strong coupling. Allowing for spatial modulation of the magnetization, we find that an inhomogeneous magnetic phase preempts the transition into the uniform phase. The resulting textured phase has similar extent and wave vector to the analytical prediction and slightly penetrates the uniform phase, lending support to the conclusions of the perturbative field theoretic analysis.

In conclusion, quantum fluctuations are known to drive the itinerant ferromagnetic transition first order. This same

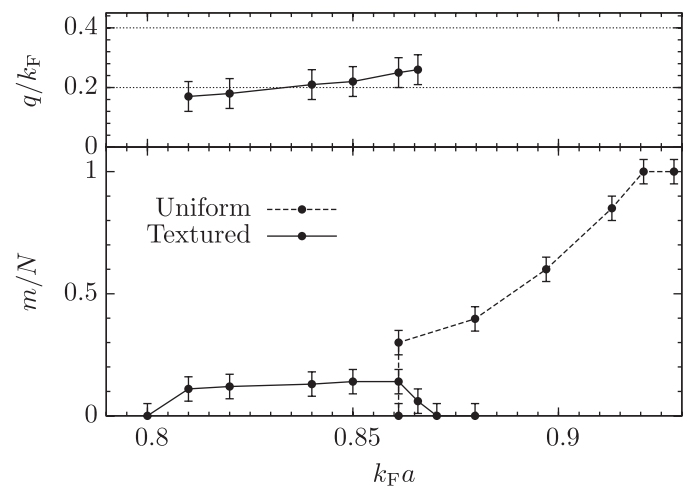

FIG. 2. The lower panel shows the variation of the ground state magnetization $m$ with interaction strength $k_{F} a$ at zero temperature. The dashed line corresponds to the uniform phase, and the solid line is the textured phase. The upper panel shows the ground state wave vector of the inhomogeneous magnetic phase, estimated from results at three discrete values of $q$ highlighted by the horizontal dotted lines. 
mechanism leads to the development of inhomogeneous magnetic order in the vicinity of the tricritical point, resulting in a phase diagram which mirrors that of the superconducting FFLO phase. Our results are consistent with recent diagrammatic analyses and reveal the connection between these works [12] and older second order perturbation calculations [14]. Moreover, our approach represents a considerable analytical simplification and more clearly reveals the underlying physical processes. This motivates searching for similar phenomena in itinerant antiferromagnetic systems as well as the possibility of a fluctuationdriven FFLO at zero magnetic field in $d$-wave superconductors.

There are several directions in which this analysis might be extended. An electronic band dispersion can drive a similar reconstruction of the electronic phase diagram [27]. It would be informative to investigate the interplay between such effects and quantum fluctuations. As well as the possibility of spatially modulated magnetism, recent works have suggested that a band dispersion might lead to $d$-wave distortion of the Fermi surface [28]. Such electron nematics may be viewed as melted versions of spatially modulated magnetism. Indeed, one might envisage a variety of spin crystalline phases and their melted nematics, driven by quantum fluctuations and occupying the same broad region of the phase diagram as studied here.

Finally, we comment upon the possibility of observing these effects experimentally. A spatially modulated magnetic phase would have its clearest signature in elastic neutron scattering, but possibly also in anisotropic transport and nuclear magnetic resonance. Determining whether such modulations are driven by quantum fluctuations or by electronic band dispersions is difficult. We note, however, that the topology of the latter case is different from that studied here [27]. Although the antiferromagnetic behavior observed in $\mathrm{NbFe}_{2}$ [9] and $\mathrm{UGe}_{2}$ [7] is consistent with magnetic texturing, a definitive identification of inhomogeneous phase formation is still lacking.

We thank Andrew Berridge, Joseph Betouras, Andrei Chubukov, Una Karahasanovic, and Andrew Schofield for useful discussions. Neil Drummond and Zoltan Radnai provided invaluable assistance with the QMC calculations. The authors acknowledge the financial support of the EPSRC, the Royal Society, and the Miller Institute.

*gjc29@cam.ac.uk

[1] J. A. Hertz, Phys. Rev. B 14, 1165 (1976); A. J. Millis, Phys. Rev. B 48, 7183 (1993); T. Moriya, Solid State Science (Springer, Berlin, Heidelberg, 1985), Vol. 56.

[2] P. Coleman and A. J. Schofield, Nature (London) 433, 226 (2005).

[3] M. Uhlarz, C. Pfleiderer, and S. M. Hayden, Phys. Rev. Lett. 93, 256404 (2004).

[4] A. Huxley, I. Sheikin, and D. Braithwaite, Physica B (Amsterdam) 284-288, 1277 (2000).
[5] C. Pfleiderer, S. Julian, and G. Lonzarich, Nature (London) 414, 427 (2001); W. Yu et al., Phys. Rev. Lett. 92, 086403 (2004).

[6] M. Otero-Leal et al., arXiv:0806.2819v1.

[7] A. Huxley et al., Phys. Rev. B 63, 144519 (2001); S. Watanabe and K. Miyake, J. Phys. Chem. Solids 63, 1465 (2002)

[8] F. Baumberger et al., Phys. Rev. Lett. 96, 107601 (2006).

[9] M. Crook and R. Cywinski, J. Magn. Magn. Mater. 140144, 71 (1995); M. Brando et al., Physica (Amsterdam) 378-380B, 111 (2006).

[10] R. Borzi et al., Science 315, 214 (2007).

[11] R. B. Laughlin et al., Adv. Phys. 50, 361 (2001).

[12] M. Shimizu, Proc. Phys. Soc. London 84, 397 (1964); D. Belitz, T. R. Kirkpatrick, and T. Vojta, Phys. Rev. B 55, 9452 (1997); J. Betouras, D. Efremov, and A. Chubukov, Phys. Rev. B 72, 115112 (2005); J. Rech, C. Pépin, and A. V. Chubukov, Phys. Rev. B 74, 195126 (2006); D. V. Efremov, J. J. Betouras, and A. Chubukov, Phys. Rev. B 77, 220401(R) (2008); D. L. Maslov and A. V. Chubukov, Phys. Rev. B 79, 075112 (2009).

[13] For a review, see D. Belitz, T. Kirkpatrick, and T. Vojta, Rev. Mod. Phys. 77, 579 (2005).

[14] A. A. Abrikosov and I. M. Khalatnikov, Sov. Phys. JETP 6, 888 (1958); F. Mohling, Phys. Rev. 122, 1062 (1961); R. A. Duine and A.H. MacDonald, Phys. Rev. Lett. 95, 230403 (2005).

[15] P. Fulde and R. A. Ferrel, Phys. Rev. 135, A550 (1964); A. I. Larkin and Y. N. Ovchinnikov, Zh. Eksp. Teor. Fiz. 47, 1136 (1964) [Sov. Phys. JETP 20, 762 (1965)].

[16] G. J. Conduit and B. D. Simons, Phys. Rev. A 79, 053606 (2009).

[17] See EPAPS Document No. E-PRLTAO-103-051943 for suupplementary material. For more information on EPAPS, see http://www.aip.org/pubservs/epaps.html.

[18] R. K. Pathria, Statistical Mechanics (ButterworthHeinemann, Oxford, Boston, 1996).

[19] The focus on nonzero temperatures leads to a regularization of the various nonanalyticities in the magnetization and its gradients, legitimizing the Landau expansion.

[20] R. Needs et al., CASINO version 2.3 User Manual (Cambridge University, Cambridge, England, 2008); W. M. C. Foulkes et al., Rev. Mod. Phys. 73, 33 (2001).

[21] D. M. Ceperley and B. J. Alder, Phys. Rev. Lett. 45, 566 (1980); G. Ortiz, M. Harris, and P. Ballone, Phys. Rev. Lett. 82, 5317 (1999); F. H. Zong, C. Lin, and D. M. Ceperley, Phys. Rev. E 66, 036703 (2002).

[22] Z. Radnai and R. Needs (unpublished).

[23] N. D. Drummond, M. D. Towler, and R. J. Needs, Phys. Rev. B 70, 235119 (2004).

[24] P. López Ríos et al., Phys. Rev. E 74, 066701 (2006).

[25] J. Carlson et al., Phys. Rev. Lett. 91, 050401 (2003).

[26] We checked that the results did not depend on different well parameters that have the same scattering length, and that a square well potential yields the same ground state energy. Results were extrapolated to zero time step, and finite size effects were reduced by twist averaging.

[27] A. M. Berridge et al., Phys. Rev. Lett. 102, 136404 (2009).

[28] H.-Y. Kee and Y. B. Kim, Phys. Rev. B 71, 184402 (2005); E. Fradkin, S. Kivelson, and V. Oganesyan, Science 315, 196 (2007). 